\title{
Constraints on Cosmic-Ray Acceleration and Transport from Isotope Observations
}

\author{
M. E. Wiedenbeck ${ }^{1}$, W. R. Binns ${ }^{2}$, E. R. Christian ${ }^{3}$, A. C. Cummings ${ }^{4}$, J. S. George ${ }^{4}$, \\ P. L. Hink ${ }^{2}$, J. Klarmann ${ }^{2}$, R. A. Leske ${ }^{4}$, M. Lijowski ${ }^{2}$, R. A. Mewaldt ${ }^{4}$, E. C. Stone ${ }^{4}$, \\ T. T. von Rosenvinge ${ }^{3}$, and N. E. Yanasak ${ }^{1}$ \\ ${ }^{I}$ Jet Propulsion Laboratory, Pasadena, CA 91109, USA \\ ${ }^{2}$ Washington University, St. Louis, MO 63130, USA \\ ${ }^{3}$ NASA/Goddard Space Flight Center, Greenbelt, MD 20771, USA \\ ${ }^{4}$ California Institute of Technology, Pasadena, CA 91125, USA
}

\begin{abstract}
Observations from the Cosmic Ray Isotope Spectrometer (CRIS) on ACE have been used to derive constraints on the locations, physical conditions, and time scales for cosmic-ray acceleration and transport. The isotopic composition of $\mathrm{Fe}, \mathrm{Co}$, and $\mathrm{Ni}$ is very similar to that of solar system material, indicating that cosmic rays contain contributions from supernovae of both Type II and Type Ia. The electron-capture primary ${ }^{59} \mathrm{Ni}$ produced in supernovae has decayed, demonstrating that a time $\gtrsim 10^{5}$ yr elapses before acceleration of the bulk of the cosmic rays and showing that most of the accelerated material is derived from old stellar or interstellar material rather than from fresh supernova ejecta.
\end{abstract}

\section{INTRODUCTION}

The Cosmic Ray Isotope Spectrometer (CRIS) instrument (1) on the Advanced Composition Explorer (ACE) has returned more than two years of isotopically-resolved measurements of the composition of low-energy galactic cosmic rays ( $\sim 50$ to $\sim 500 \mathrm{MeV} /$ nucleon) under conditions of relatively low solar modulation. Because of the instrument's large geometrical acceptance, $\sim 250 \mathrm{~cm}^{2} \mathrm{sr}$, this data set allows statistically-significant determinations of the abundances of essentially all stable and long-lived cosmic-ray isotopes from $\mathrm{He}$ to $\mathrm{Cu}$ (atomic numbers $Z=2$ to 29 ), with exploratory measurements for elements with $30 \leq Z \lesssim 34$.

These data are being used to investigate a wide range of topics. In this paper we discuss the isotopic composition of $\mathrm{Fe}, \mathrm{Co}$, and $\mathrm{Ni}$ in the cosmic-ray source and its implications for the environment from which cosmic rays are accelerated. We also examine the abundances of primary electron-capture nuclides and their daughter products and use these results to derive constraints on the time that elapses between nucleosynthesis and acceleration and on the conditions in the acceleration volume.

Additional results based on data from CRIS are discussed in other papers in this volume. The topics covered include: the cosmic-ray confinement time in the Galaxy (Yanasak et al.); the distribution of matter thicknesses traversed during transport (Davis et al.); the effects of inflight electron attachment and decay on the energy spec- tra of pure electron-capture nuclides (Niebur et al.); elemental fractionation of the cosmic-ray seed population (George et al.); contributions of special sources (particularly of ${ }^{22} \mathrm{Ne}$ ) to the source material (Binns et al.); energy spectra of major cosmic ray elements (Leske et al., Davis et al.); and solar-modulation of galactic cosmic rays on short time scales (Christian et al.).

\section{SOURCE COMPOSITION}

The composition of the source material that is accelerated to produce galactic cosmic rays (GCRs) can help in determining the objects in which the nucleosynthesis of this material occurs and the regions of the Galaxy from which particles are accelerated. This information then constrains models for the acceleration and transport of the particles. It is well established that the elemental composition of the cosmic-ray source is very similar to solar system composition, once fractionation effects related to atomic properties such as first ionization potential or volatility are taken into account (2). In addition, it has been shown that the isotopic composition of major cosmic-ray elements including $\mathrm{Mg}, \mathrm{Si}, \mathrm{Fe}$, and $\mathrm{Ni}$ does not differ markedly from solar-system (i.e., terrestrial) composition. Indeed, at present there is only one element, $\mathrm{Ne}$, with a well established isotopic difference between the cosmic-ray source and the solar system $(3,4,5,6,7)$. 

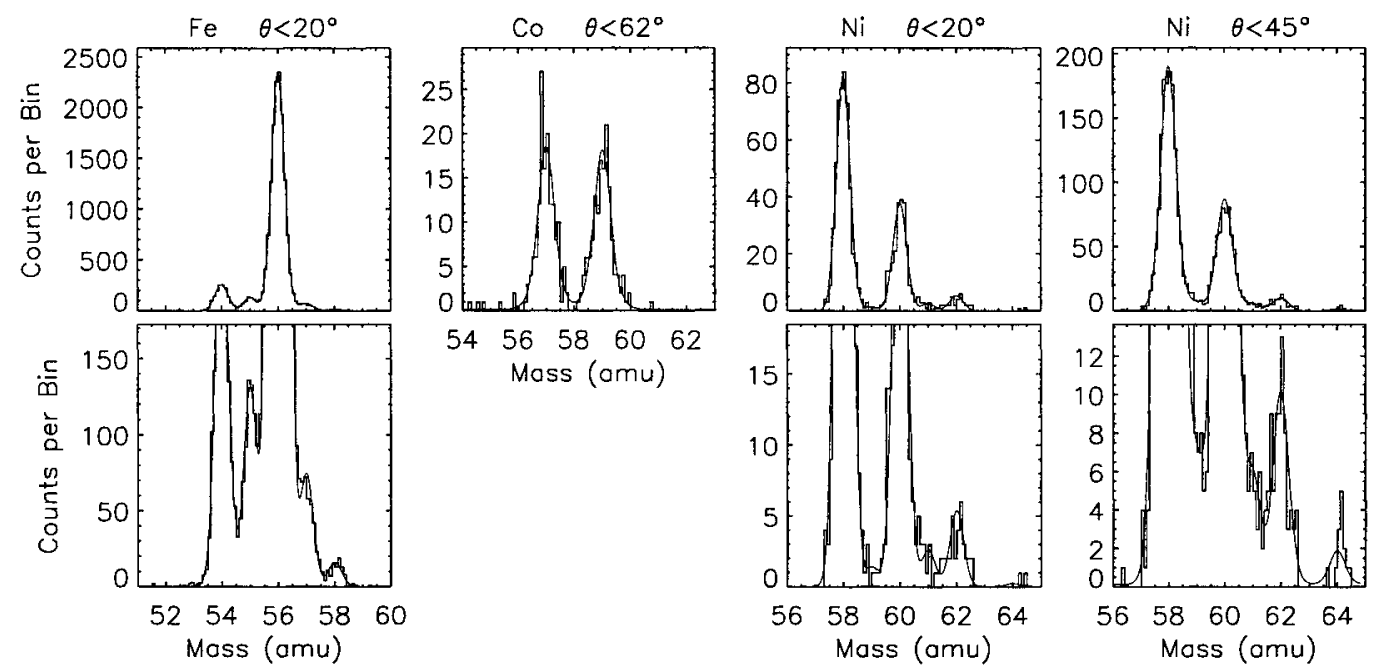

FIGURE 1. Measured mass distributions for $\mathrm{Fe}, \mathrm{Co}$, and $\mathrm{Ni}$ with selected cuts on angle of incidence, $\theta$, as indicated. The lower panels show the same histograms with vertical scales expanded to better show the rare isotopes.

\section{ACE/CRIS Observations}

Using ACE data it is possible to compare these samples of matter in greater detail by improving the statistical accuracy of the measurements and by determining abundances of very rare cosmic-ray isotopes. Of particular interest are the isotopes of $\mathrm{Fe}, \mathrm{Co}$, and $\mathrm{Ni}$. Among these three elements there are ten stable isotopes, and among them there is a general trend of decreasing abundances with increasing mass. As a result, there are only relatively minor contributions to the observed abundances of stable cosmic-ray nuclides (other than ${ }^{54} \mathrm{Fe}$ ) from fragmentation of heavier species during transport through the interstellar medium (ISM), and source abundances can be derived with reasonable accuracy.

Figure 1 shows mass histograms for $\mathrm{Fe}, \mathrm{Co}$, and $\mathrm{Ni}$ obtained from CRIS data collected between August 1997 and December 1998. The plots in the upper row of the figure show the complete histograms while those in the lower row show the same data with the vertical scales expanded to better show the rare isotopes. For Ni, two sets of histograms are shown differing in the maximum angle $(\theta$, measured from the detector normal) to which particles were accepted. The rms mass resolution for iron-group isotopes is $0.23 \mathrm{amu}$ at small angles of incidence and has an approximately parabolic dependence on $\theta$, increasing to about twice the $\theta=0^{\circ}$ value for $\theta \simeq 45^{\circ}$. By considering only events with $\theta$ less than some appropriatelychosen maximum value, it is possible to optimize the trade-off between resolution and statistics for the isotopes of interest. Thus, in the $\theta<20^{\circ}$ histogram, the spill-over into the ${ }^{59} \mathrm{Ni}$ region from the abundant adjacent isotopes is minimized, while in the $\theta<45^{\circ}$ histogram the rare isotope ${ }^{64} \mathrm{Ni}$ is present with reasonable statistics. On the histograms, fits to the mass distributions are shown. For each isotope the fitted peak shape is a superposition of Gaussians with a distribution of widths corresponding to the angular distribution of particles in the data set, and it has been assumed that the shape is the same for all isotopes of an element. For Co there is negligible overlap between the two isotope peaks so the abundances were obtained by event counting rather than from the fits.

To obtain isotopic abundances for an element, the measured relative numbers of events were corrected for differences in energy interval and differences in the probability for loss by nuclear interaction in the CRIS instrument. These corrections are relatively small, amounting to $6.6 \%$ and $1.5 \%$, respectively, in the most extreme case $\left({ }^{64} \mathrm{Ni} /{ }^{58} \mathrm{Ni}\right)$.

The elemental abundances are derived by calculating energy spectra for a wide range of elements, fitting that set of spectra with a common shape, and obtaining the relative abundances from the normalization factors for the various spectra. Figure 2 shows the measured spectra for $\mathrm{Fe}, \mathrm{Co}$, and $\mathrm{Ni}$, along with the fits.

To obtain the cosmic-ray source composition from the measurements, a leaky-box propagation model was used to estimate the required secondary corrections to the measured abundances. The model parameters were taken from (8). The solar modulation level was modified to correspond to the near-solar-minimum conditions under which the CRIS data were collected (modulation parameter $\phi \simeq 500 \mathrm{MV}$ ). This model adequately accounts for a variety of secondary nuclides in the sub-iron region, $\mathrm{Sc}$ through $\mathrm{Mn}$.

For the present discussion we consider isotopes with mass number $A \geq 56$ and do not present a source abundance for ${ }^{54} \mathrm{Fe}$. With the exception of ${ }^{56} \mathrm{Fe}$, this nuclide 


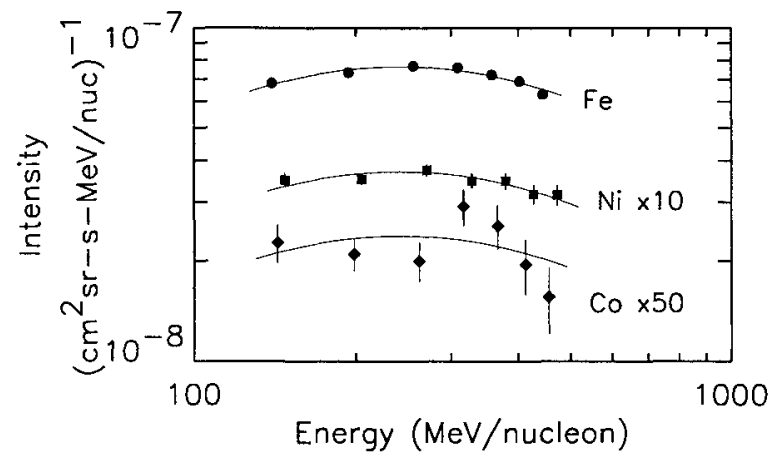

FIGURE 2. Energy spectra of Fe, Co, and Ni measured with CRIS. Error bars indicate statistical uncertainties only and are smaller than the plotted points in some cases.

has the largest source abundance among the isotopes of $\mathrm{Fe}, \mathrm{Co}$, and $\mathrm{Ni}$ and it has been reported (5) that the ${ }^{54} \mathrm{Fe} /{ }^{56} \mathrm{Fe}$ source ratio is enhanced by $\sim 50 \%$ over the solar value. The derivation of the ${ }^{54} \mathrm{Fe}$ source abundance is complicated by a significant secondary contribution from fragmentation of ${ }^{56} \mathrm{Fe}$ (directly and through ${ }^{55} \mathrm{Fe}$ ) and by the fact that a fraction of the secondary ${ }^{54} \mathrm{Mn}$ produced during propagation decays by $\beta^{-}$emission to ${ }^{54} \mathrm{Fe}(9,8)$. CRIS results on the ${ }^{54} \mathrm{Fe}$ source abundance will be reported after a self-consistent analysis of these effects is carried out.

\section{Derived Source Abundances}

Figure 3 compares the cosmic-ray source abundances we obtain with those found in solar system material (10). Abundances in each sample have been normalized so that ${ }^{56} \mathrm{Fe} \equiv 1$. As seen in the figure, there is a strong correlation between the abundances of the $\mathrm{Fe}, \mathrm{Co}$, and $\mathrm{Ni}$ isotopes in the two populations, even though the relative abundances range over a factor of $\sim 2000$. Abundance differences for individual isotopes are less than a factor of 2, and commonly no more than a few tens of percent. This result is in line with recent reports $(3,4,6)$ that the abundances of the isotopes of $\mathrm{Mg}$ and $\mathrm{Si}$ are in good agreement with solar system values, typically differing by $\lesssim 20 \%$.

The great similarity between GCR source and solar system compositions is all the more striking when compared with abundance distributions expected from various stellar nucleosynthesis models. Attempts to account for solar system abundances as sums of the ejecta from supernovae of various types and initial masses have found $(11,12)$ that contributions are required both from Type II supernovae (hereafter, SN II), which result from the core collapse of massive stars $\left(\gtrsim 8 M_{\odot}\right)$, and Type Ia supernovae (SN Ia), which are caused by the accretion of a

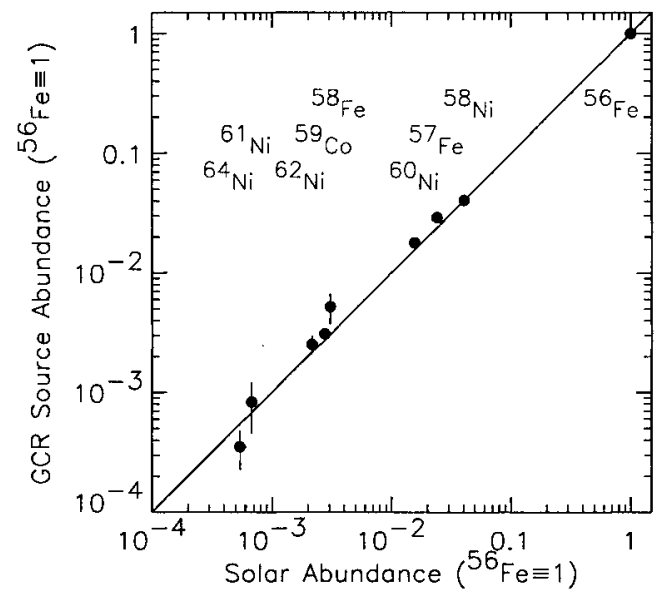

FIGURE 3. Comparison of GCR source abundances with solar system values.

critical mass of matter onto white dwarf stars in binary systems. Whereas the abundances of intermediate-mass elements such as $\mathrm{Mg}$ and $\mathrm{Si}$ can be reasonably explained in terms of SN II contributions alone, the abundances of iron-group elements are not adequately explained in this way (13). Rather, one finds that a mixture in which $\sim 10 \%$ if the mass comes from SN Ia and the other $\sim 90 \%$ from SN II can account for the iron-group nuclides (11). Because of the preponderance of iron-group material in SN Ia ejecta, these explosions would provide $\gtrsim 50 \%$ of the $\mathrm{Fe}$ and $\mathrm{Ni}$ by contributing only $\sim 10 \%$ of the total mass.

In addition to comparisons such as $\mathrm{Fe}+\mathrm{Ni}$ versus $\mathrm{Mg}+\mathrm{Si}$, one can also consider the origins predicted for specific nuclides in the various nucleosynthesis models. For example, the SN II yield calculations of Thielemann et al. (14) indicate that very neutron-rich nuclides such as ${ }^{58} \mathrm{Fe}$ and ${ }^{64} \mathrm{Ni}$ should be nearly absent in the ejecta. They attribute the origin of these species to production in SN Ia. Alternative SN II calculations by Woosley and Weaver (15) do not confirm this deficit of very neutronrich species but do indicate that $\mathrm{SN}$ II can only produce sufficient ${ }^{58} \mathrm{Fe}$ in stars more massive than $15 M_{\odot}$, while these massive stars systematically over-produce ${ }^{64} \mathrm{Ni}$. It is clear that differences among available supernova yield calculations dictate caution in using them for interpreting detailed abundance observations. Indeed, we find that the agreement between the isotopic composition of the cosmic-ray source (with the exception of ${ }^{22} \mathrm{Ne}$ ) and that of the solar system is generally better than the agreement among available supernova yield calculations by different research groups.

The similarity between the GCR source and solar system compositions may arise naturally if cosmic rays are accelerated out of the general interstellar medium and if 
there has been relatively little evolution of the composition of that medium during the 4.5 Gyr since the solar system condensed out of it. Two scenarios of this type have been widely discussed: first, direct acceleration from the gas and dust in the general ISM and second, acceleration of material from the coronae of stars.

In the first class of models, the acceleration mechanism must be capable of injecting atoms from grains with comparable efficiency to injection from the gas phase, since refractory elements are largely depleted from the gas phase of the ISM and reside in grains. In fact, the grain material should be accelerated with greater efficiency than gas-phase material by the factor $\sim 5$ found for the relative fractionation of high- and low-volatility elements in the GCR source (16). Theoretical work has led to models (17) in which interstellar shocks efficiently accelerate charged grains to relatively high velocities where ions are sputtered from the grains and injected into the shock for acceleration along with the gas-phase ions.

In the second class of models, the initial step in the energization of the material occurs in stellar coronae where flares and CME-driven shocks analogous to those from the Sun produce particles in the $\mathrm{MeV}$ energy range $(18,19)$. This energetic material is subsequently further accelerated by shocks in the ISM. Since the composition of matter at the surfaces of most stars remains essentially unaltered from the composition of the material from which those stars formed, one expects abundances characteristic of the average ISM, including both gas and dust components. This scenario has the attractive feature that it accounts for the observed elemental fractionation of the GCR source as due to the same mechanism, presumably related to first ionization potential or first ionization time, that produces the observed fractionation of the solar wind and solar energetic particles. However, there remains an unresolved problem of how the stellar ejecta can remain energetic long enough to encounter the shocks needed for acceleration to cosmic-ray energies.

The cosmic-ray data provide observational constraints which must be accounted for by any successful model of cosmic-ray origin and acceleration. The need for significant contributions of material from both SN II and SN Ia restricts models which involve acceleration of material from a limited portion of the ISM. Stars form preferentially in associations as giant molecular clouds collapse. The more massive of the stars evolve rapidly and undergo supernovae explosions while still closely grouped. The evolutionary time scales for low-mass stars are much longer and these stars survive long after the original associations have lost their identity and the stars have been spread extensively into the Galaxy. Thus contributions of material from SN Ia should be broadly and rather smoothly distributed through the Galaxy, not clustered like the SN II contributions.
It has been proposed (20) that cosmic rays are preferentially accelerated in super-bubbles produced as winds and explosions of massive stars in associations blow hot, low-density voids in the general ISM. For such a model to account for the observed GCR source composition requires that this hot, tenuous phase of the ISM occupy a large fraction of galactic volume so that SN Ia will be sufficiently abundant (relative to SN II) in the acceleration volume. It is thought that this fraction is $\sim 50 \%$ averaged over the Galaxy's volume (see, for example, (20) and references therein), and possibly a significantly larger fraction inside the solar system's galactocentric radius (21).

In addition to their significance for understanding the origin of galactic cosmic rays, the GCR source isotopic abundances being obtained from CRIS are directly relevant to understanding the composition of present-day interstellar matter and the rate of chemical evolution in the Galaxy. Isotopic (as opposed to elemental) abundance determinations in material outside the solar system are presently limited to a relatively few elements, primarily those that form interstellar gas molecules with rotational and/or vibrational transitions that can be used to distinguish the mass of the atoms involved by spectroscopic means (22). For most elements, and particularly for noble gases and refractory elements that are depleted from the gas phase, very little is known about isotopic composition. (See, however, (23) concerning the isotopic composition of $\mathrm{Ne}$ in the very local ISM as derived from studies of the anomalous component of cosmic rays.)

It can be argued that the GCR source composition presently provides the best estimate of the isotopic composition of certain important elements in the ISM, including $\mathrm{Mg}, \mathrm{Si}, \mathrm{Fe}, \mathrm{Ni}, \mathrm{Cu}$, and $\mathrm{Zn}$. Further analysis of data from CRIS should add several elements to this list. Uncertainties remain concerning the details of the location and physical conditions of the galactic material from which cosmic rays are derived, but these appear no more limiting than those which are encountered in attempting to extract overall galactic isotopic composition from measurements of interstellar molecules. In fact, comparison of isotopic composition results for elements such as $\mathrm{C}$, $\mathrm{O}$, and $\mathrm{S}$ which can be investigated by both techniques should be useful for assessing the magnitude of these uncertainties.

\section{ACCELERATION CONSTRAINTS FROM ELECTRON-CAPTURE PRIMARIES}

Additional constraints on the location and nature of cosmic-ray acceleration are related to the time that elapses between nucleosynthesis of the cosmic-ray seed material and acceleration to high energies. It is generally accepted that the major contributors to cosmic-ray 
acceleration in the Galaxy are shocks produced by supernovae. This view is supported both by the fact that supernovae are the only objects that are known to output sufficient power to sustain the observed GCR intensity and by the fact that observations of synchrotron radiation from supernova remnants show that at least electrons have been accelerated to relativistic energies there. However, a long-standing question is whether the bulk of the material accelerated by supernova shocks originates from the ejecta of the same exploding star or from surrounding interstellar matter.

It was pointed out by Soutoul et al. (25) that this elapsed time can be derived from the observed abundances of radioactive cosmic-ray nuclides that should be produced predominantly in supernovae explosions (rather than as secondaries from fragmentation during transport through the Galaxy) and that can decay only by orbital electron capture. After ejection by the explosion, nuclei reside in the thermal matter of the remnant for an unknown time before acceleration occurs. Under these conditions, electron-capture decays proceed with the laboratory halflife. Once acceleration occurs and a small amount of matter has been traversed at high energies (a few tens of $\mathrm{mg} / \mathrm{cm}^{2}$ is sufficient), the nuclei become stripped of their orbital electrons and are effectively stable. Thus the presence (absence) of a significant primary component of an electron-capture nuclide in the cosmic rays that are observed indicates an elapsed time between nucleosynthesis and acceleration which is short (long) compared to the halflife.

\section{Acceleration Time Delay}

A sizeable number of pure-electron-capture nuclides are observed with CRIS, but most of these are dominated by secondaries produced during propagation. While of interest for studying processes occurring during transport, these secondary-dominated nuclides are not useful for investigating the acceleration time delay. Two electroncapture nuclides, ${ }^{59} \mathrm{Ni}\left(T_{1 / 2}=7.6 \times 10^{4} \mathrm{yr}\right)$ and ${ }^{57} \mathrm{Co}$ $\left(T_{1 / 2}=0.74 \mathrm{yr}\right)$, have small secondary contributions because they are too massive to be produced by fragmentation of abundant cosmic-ray species such as ${ }^{56} \mathrm{Fe}$. As seen in Figure $1,{ }^{59} \mathrm{Ni}$ appears to be absent, or nearly so, while its stable daughter product ${ }^{59} \mathrm{Co}$ is clearly present.

The upper two panels of Figure 4 show how the observed abundances of the mass-59 isotopes would vary depending on the delay time between nucleosynthesis and acceleration. The observed abundances contain contributions from both secondaries (dashed lines) and primaries. The primary ${ }^{59} \mathrm{Ni}+{ }^{59} \mathrm{Co}$ is constrained by the observed abundances, but the fraction $f_{0}=$ $\left({ }^{59} \mathrm{Ni} /\left({ }^{59} \mathrm{Ni}+{ }^{59} \mathrm{Co}\right)\right)_{\text {primary }}$ is not. The various solid

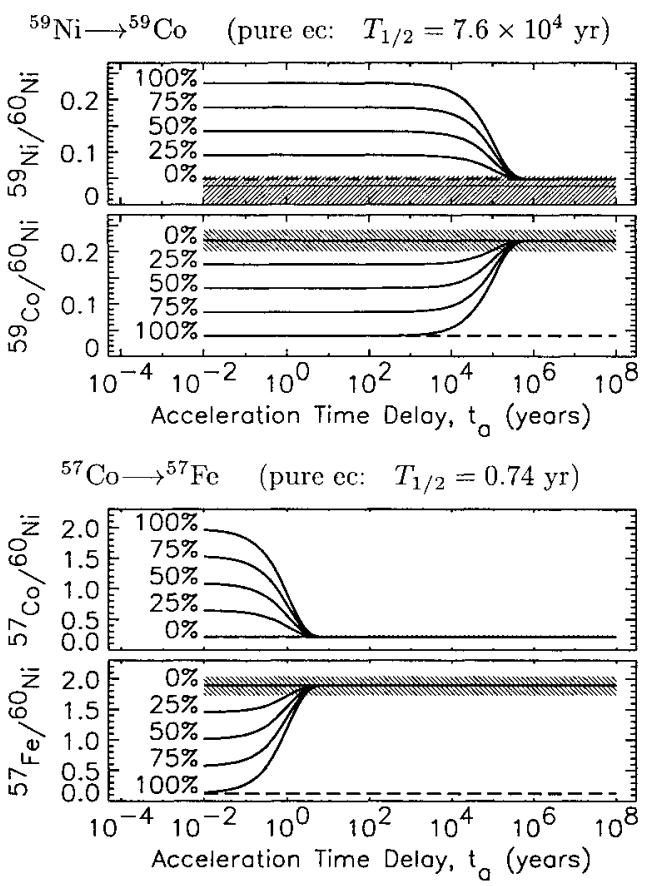

FIGURE 4. Dependence of arriving GCR abundances of electron-capture nuclides and their daughters on the time delay between nucleosynthesis and acceleration. (See description in the text.)

curves are parameterized by the value of $f_{0}$, which represents the fraction of the primary mass-59 material synthesized in the form of ${ }^{59} \mathrm{Ni}$. The hatched regions show the observed abundances from CRIS with their one standard deviation uncertainties. The CRIS observations, which indicate that in the arriving cosmic rays all of the mass59 primaries are in the form of ${ }^{59} \mathrm{Co}$, are consistent with either a time delay before acceleration which is long compared to the $7.6 \times 10^{4} \mathrm{yr}$ halflife of ${ }^{59} \mathrm{Ni}$, or a nucleosynthesis process which produces ${ }^{59} \mathrm{Co}$ rather than ${ }^{59} \mathrm{Ni}$.

To distinguish between these two possibilities one can examine theoretical predictions for the relative yields of the ${ }^{59} \mathrm{Co}$ and ${ }^{59} \mathrm{Ni}$ from supernova explosions. Figure 5 shows the allowed combinations of the delay time, $t_{a}$, and the ${ }^{59} \mathrm{Ni}$ fraction, $f_{0}$, as a $98 \%$ confidence region (hatched area). The dashed lines show the minimum, maximum, and average (weighted by a Salpeter initial mass function [IMF]) values of $f_{0}$ found from numerical calculations (15) of ejected yields from SN II resulting from stars of various initial masses. Other calculations (12) indicate that ${ }^{59} \mathrm{Ni}$ should constitute a major fraction of the mass59 ejecta from SN Ia. Thus there should have been a significant initial fraction of ${ }^{59} \mathrm{Ni}$ from any combination of SN II and SN Ia sources, and one can conclude that a delay time $\gtrsim 10^{5}$ yr between nucleosynthesis and accel- 
eration is needed to account for the CRIS observations (26).

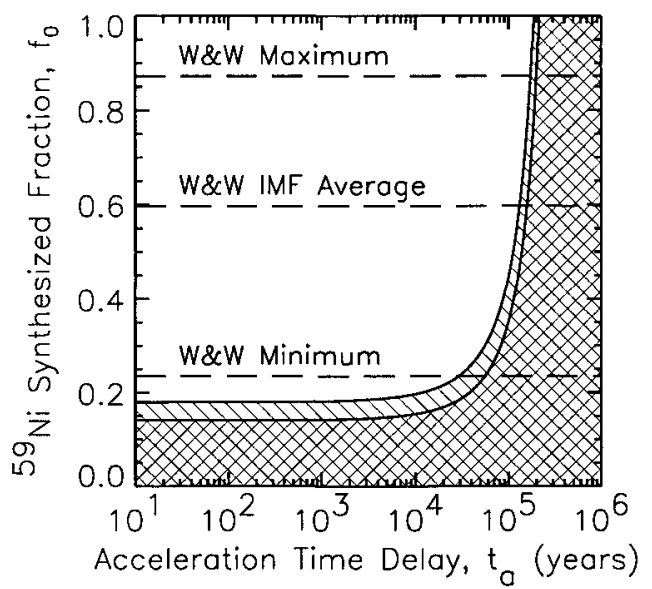

FIGURE 5. Allowed combinations (98\% confidence) of acceleration delay times and fractions of mass-59 material synthesized as ${ }^{59} \mathrm{Ni}$. The cross-hatched region is based on the CRIS measurement uncertainties alone while the diagonally-hatched extension also takes into account estimated cross-section uncertainties.

This time delay before acceleration is significantly longer than the time required for a supernova shock to dissipate its energy (27), so we conclude that the seed population for cosmic-ray acceleration is predominantly old stellar or interstellar material rather than fresh supernova ejecta. This is consistent with the above results (Fig. 3) which show that the isotopic composition of the GCR source is very similar to that of the solar system. To obtain such a result in a model where supernovae promptly accelerate a portion of their own ejecta would require that acceleration efficiencies be comparable over a sizeable range of stellar masses so that the cosmic-ray source would contain a mix of contributions similar to that which produced abundances found in the general ISM.

To be consistent with the mass-59 observations, one expects that all of the observed mass- 57 primaries should be in the form of the daughter product, ${ }^{57} \mathrm{Fe}$, rather than the parent ${ }^{57} \mathrm{Co}$, since the halflife of the latter nuclide $(0.74 \mathrm{yr})$ is much shorter than the halflife of ${ }^{59} \mathrm{Ni}$. The lower two panels of Figure 4 show how the observed abundances of the mass- 57 nuclides should depend on the time between nucleosynthesis and acceleration, assuming that the ${ }^{57} \mathrm{Co}$ is stable after acceleration. (As will be discussed below, this assumption will be valid only under a limited set of acceleration conditions.) As expected, the ${ }^{57} \mathrm{Co}$ and ${ }^{57} \mathrm{Fe}$ data are consistent with complete decay of primary ${ }^{57} \mathrm{Co}$.

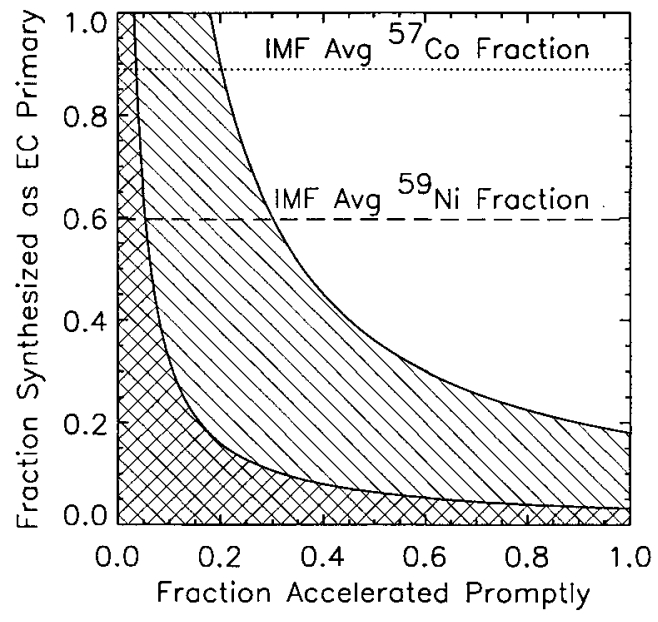

FIGURE 6. Fractions of the cosmic rays that could have been accelerated promptly compared to the halflives of ${ }^{59} \mathrm{Ni}$ ( $\sim 10^{5} \mathrm{yr}$, diagonal hatching) and ${ }^{57} \mathrm{Co}(\sim 1 \mathrm{yr}$, cross hatching). The hatched regions correspond to $98 \%$ confidence levels and take into account both the CRIS measurement errors and estimated uncertainties on the fragmentation cross sections.

\section{Fraction Accelerated Promptly}

In the above discussion it was assumed that all of the cosmic rays are accelerated with a common delay time between nucleosynthesis and acceleration. In reality, one should expect some distribution of delay times, and the observed abundances of electron-capture primaries can be used to provide information about this distribution. For simplicity we assume that the accelerated material can be divided into two populations: a "prompt" population for which the time delay was much less than the halflife of the isotope being considered and a "delayed" population accelerated on times scales much longer than the halflife. From the observational upper limits on the abundances of the electron-capture primaries one can set upper limits on the fraction of material in the prompt category. This upper limit also depends on $f_{0}$, the fraction of the material of the particular mass number synthesized as the electron-capture primary (rather than as its daughter product). Figure 6 shows $98 \%$ confidence regions indicating the fractions of the material accelerated promptly compared to the $7.6 \times 10^{4}$ yr halflife of ${ }^{59} \mathrm{Ni}$ (diagonal hatching) and compared to the $0.74 \mathrm{yr}$ halflife of ${ }^{57} \mathrm{Co}$ (cross hatching).

Also shown are dashed and dotted lines indicating the values of $f_{0}$ predicted for ${ }^{59} \mathrm{Ni}$ and ${ }^{57} \mathrm{Co}$, respectively, by taking IMF-weighted averages of the calculated yields from the SN II models of Woosley and Weaver (15). From the ${ }^{59} \mathrm{Ni}$ result we conclude that up to $\sim 30 \%$ of the cosmic-rays could have been accelerated on time scales $\ll 10^{5}$ yr without causing a significant conflict with the 
observed composition. The ${ }^{57}$ Co results potentially provide a more stringent limit $(\lesssim 5 \%)$ on the fraction accelerated on time scales $\ll 1$ yr. However, the applicability of the ${ }^{57} \mathrm{Co}$ results depends strongly on the physical environment in which the acceleration occurs since this influences the chances of in-flight electron attachment and subsequent decay. This problem is discussed in the next subsection.

\section{Electron Attachment and Loss in Flight}

In the simple interpretations presented above of the abundances of primary electron-capture nuclides and their daughters, the assumption was made that cosmic-ray acceleration occurs over a time interval short compared to the $\sim 2 \times 10^{7} \mathrm{yr}$ confinement time in the Galaxy (28) and that during acceleration and propagation the nuclei remain fully stripped of any electrons.

However, the probability for electron attachment in flight is not completely negligible and can affect the interpretation of the observed abundances of electron-capturedecay nuclides. The upper panel in Figure 7 shows, as a function of energy per nucleon, the mean amounts of interstellar matter (assumed to be composed only of $\mathbf{H}$ and $\mathrm{He}$ in a 10:1 ratio by number) that a Co nucleus must traverse to attach an electron or to strip off an electron that was previously attached (29). In the lower panel the corresponding mean times for attachment and stripping are shown, assuming a medium with a uniform density of $0.25 \mathrm{H}$ atoms $/ \mathrm{cm}^{3}$, as indicated by the surviving fractions of $\beta$-unstable secondaries such as ${ }^{10} \mathrm{Be}(28)$.

Energetic ${ }^{57} \mathrm{Co}$ ions passing through interstellar matter will tend to be stripped of their electrons in passing through $\sim 1-10 \mathrm{mg} / \mathrm{cm}^{2}$ of material (Fig. 7). If this matter is traversed on a time scale short compared to the $\sim 0.74$ yr ${ }^{57} \mathrm{Co}$ halflife, decays will be prevented. This will be the case if the acceleration occurs in a medium having a density of at least several thousand atoms $/ \mathrm{cm}^{3}$.

As shown in Figure 7, the mean free path for electron attachment by a bare Co nucleus at $600 \mathrm{MeV} /$ nucleon (the approximate interstellar energy of the particles measured with CRIS) is $\sim 11 \mathrm{~g} / \mathrm{cm}^{2}$. This is long compared to the mean free path for fragmentation of the nuclei in collisions with interstellar matter $\left(\sim 2-3 \mathrm{~g} / \mathrm{cm}^{2}\right)$, so the accelerated population of ${ }^{57} \mathrm{Co}$ will only undergo minor alteration by subsequent electron attachments followed by electron-capture decays. Thus the simple interpretation of the ${ }^{57} \mathrm{Co}$ observations presented in the previous section is applicable only if the cosmic-ray acceleration occurs in a medium dense enough to strip the nuclei before electron capture decays can occur.

One can also examine the effects of in-flight electron attachment and loss on the interpretation of the mea-

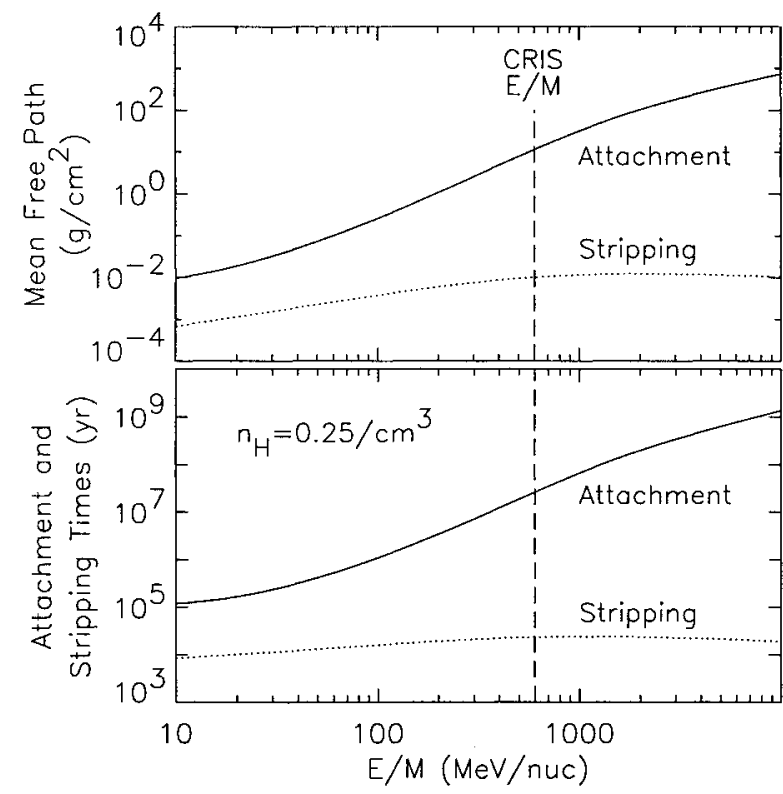

FIGURE 7. Electron attachment and loss mean free paths (29) and related quantities as a function of the energy of cosmic-ray Co nuclei. An interstellar $\mathrm{He} / \mathrm{H}$ ratio of 0.1 by number has been assumed. Dashed vertical lines indicate approximate interstellar energy of Co nuclei measured by CRIS.

sured ${ }^{59} \mathrm{Ni}$ and ${ }^{59} \mathrm{Co}$ abundances. The mean free paths and related quantities shown for $\mathrm{Co}$ in Figure 7 differ little from the corresponding quantities for $\mathrm{Ni}$ since the high-energy attachment and loss cross sections should be smooth functions of the atomic number of the energetic nucleus. Because the halflife of ${ }^{59} \mathrm{Ni}$ is a factor of $10^{5}$ longer than that of ${ }^{57} \mathrm{Co}$, the required density to prevent decay during acceleration is smaller by this factor, amounting to just a few hundredths of an atom $/ \mathrm{cm}^{2}$. Since this value is small compared to the average density encountered by the cosmic rays during propagation, this constraint should easily be met. Thus the conclusions presented based on the decay of ${ }^{59} \mathrm{Ni}$ to ${ }^{59} \mathrm{Co}$ should be relatively robust.

To construct a scenario that would allow the in-flight decay of ${ }^{59} \mathrm{Ni}$, it is necessary to assume that particle acceleration occurs over a relatively long time span. At energies $\lesssim 150 \mathrm{MeV} /$ nucleon the electron-attachment mean free path is shorter than the mean free paths for nuclear interaction losses and for escape from the Galaxy. If the ${ }^{59} \mathrm{Ni}$ remain below this energy for a time long compared to their halflife, electron attachment and subsequent decay will convert the nuclei to ${ }^{59} \mathrm{Co}$. Although at present there is no observational indication that a slow acceleration of this sort was involved in producing galactic cosmic rays, it is also difficult to completely rule out. 


\section{CONCLUSIONS}

Detailed examination of the abundances of stable isotopes of $\mathrm{Fe}, \mathrm{Co}$, and $\mathrm{Ni}$ reveals a composition pattern which is strikingly similar to that of solar system material, differing by at most several tens of percent. This confirms and extends previous findings that the isotopic composition of other major elements, most notably $\mathrm{Mg}$ and $\mathrm{Si}$, differs little from solar system composition. These observations are in striking contrast to the isotopic composition of GCR Ne $(3,4,6)$ which is strongly enhanced in ${ }^{22} \mathrm{Ne}$ relative to the composition of the solar wind and the composition inferred for the very local ISM from observation of the anomalous cosmic-ray component of Ne. The GCR source composition can not be accounted for as a superposition of ejecta from SN II alone but appears to require contributions from $\mathrm{SN}$ Ia, as previously noted for solar system material. The requirement that the cosmicray source material contain the appropriate mix of contributions from SN II, which occur preferentially in associations, and SN Ia, which tend to be rather smoothly distributed in the Galaxy, provides a potentially useful constraint on the sites from which cosmic rays could originate.

Measurements of mass-59 nuclides in cosmic rays show that the pure electron-capture nuclide ${ }^{59} \mathrm{Ni}$ synthesized in supernovae has decayed. The simplest interpretation of this result leads to the conclusion that at least $\sim 10^{5} \mathrm{yr}$ elapses before acceleration of the bulk of the cosmic-ray material. An upper limit $\sim 30 \%$ can be set on the fraction of the material which could have been accelerated on shorter time scales.

These observations can be accounted for in a model in which cosmic rays are accelerated by supernovaproduced shocks as they propagate through the general interstellar medium accelerating a mix of nuclei previously synthesized and returned to the ISM by stars with a broad distribution of masses and corresponding evolutionary time scales, similar to the distribution that produced the material from which the solar system condensed. The population of material accelerated by supernova shocks contains at most minor contributions promptly accelerated from the ejecta driving the shocks.

\section{ACKNOWLEDGMENT}

This research was supported by the National Aeronautics and Space Administration at the California Institute of Technology (under grant NAG5-6912), the Jet Propulsion Laboratory, the Goddard Space Flight Center, and Washington University.

\section{REFERENCES}

1. Stone, E. C. et al., Sp. Sci. Rev. 86, 283 (1998).

2. Engelmann, J. J. et al., Astron. Ap. 233, 96 (1990).

3. DuVernois, M. A. et al., Ap. J. 466, 457 (1996).

4. Webber, W. R., Lukasiak, A., and McDonald, F. B., Ap. J. 476, 766 (1997).

5. Connell, J. J. and Simpson, J. A., Ap. J. Lett. 475, L61 (1997).

6. Connell, J. J. and Simpson, J. A., Proc. 25th Internat. Cosmic Ray Conf. (Durban) 3, 381 (1997).

7. Thayer, M. R., Ap. J. 482, 792 (1997).

8. Leske, R.A., Ap. J. 405, 567 (1991).

9. Grove, J. E. et al., Ap. J. 377, 680 (1991)

10. Anders, E. and Grevesse, N., Geoch. Cosmoch. Acta 53, 197 (1989).

11. Tsujimoto, T. et al., Mon. Not. Royal Astron. Soc. 277, 945 (1995).

12. Iwamoto, K. et al., Ap. J. Suppl. 125, 439 (1999).

13. Meyer, J.-P. and Ellison, D. C., in $L i B e B$, Cosmic Rays, and Related X-and Gamma-Rays, edited by R. Ramaty et al., Astro. Soc. Pacific, San Francisco, ASP Conf. Series vol. 171, 1999, pp. 187-206.

14. Thielemann, F.-K., Nomoto, K., and Hashimoto, M., Ap. J. 408 (1996)

15. Woosley, S. E. and Weaver, T. A., Ap. J. Suppl. 101, 181 (1995).

16. Meyer, J.-P., Drury, L. O'C., and Ellison, D. C., Ap. J. 487, 182 (1997)

17. Ellison, D. C., Drury, L. O'C., and Meyer, J.-P., Ap. J. 487, 197 (1997).

18. Meyer, J.-P., Ap. J. Suppl. 57, 173 (1985).

19. Shapiro, M. M., in LiBeB, Cosmic Rays, and Related Xand Gamma-Rays, edited by R. Ramaty et al., Astro. Soc. Pacific, San Francisco, ASP Conf. Series vol. 171, 1999, pp. 138-145.

20. Higdon, J.C., Lingenfelter, R.E., and Ramaty, R., Ap. J. Lett. 509, L33 (1998).

21. Lingenfelter, R. E., Higdon, J. C., and Ramaty, R. "Cosmic Ray Acceleration in Superbubbles and the Composition of Cosmic Rays", in Acceleration and Transport of Energetic Particles Observed in the Heliosphere, edited by R. Mewaldt et al., New York, AIP Conf. Proceedings, 2000 (this volume).

22. Wilson, T. L., Reports on Prog. in Phys. 62, 143 (1999).

23. Leske, R. A. et al., Sp. Sci. Rev. 78, 149 (1996).

24. Woosley, S. E. and Weaver, T. A., Ap. J. 243, 651 (1981).

25. Soutoul, A., Cassé, M., and Juliusson, E., Ap. J. 219, 753 (1978).

26. Wiedenbeck, M. E. at al., Ap. J. Lett. 523, L61 (1999).

27. Longair, M. S., High Energy Astrophysics, vol. 2, Cambridge Univ. Press, Cambridge, England, 1994.

28. Connell, J. J., Ap. J. Lett. 501, L59 (1998).

29. Crawford, H. J., Ph.D. thesis, Univ. of Calif. Berkeley, Lawrence Berkeley Laboratory Report LBL-8807 (1979). 